\title{
Serovar Determination and Molecular Taxonomic Correlation in Mycobacterium avium, Mycobacterium intracellulare, and Mycobacterium scrofulaceum: a Cooperative Study of the International Working Group on Mycobacterial Taxonomy
}

\author{
L. G. WAYNE, ${ }^{1}$ R. C. GOOD,${ }^{2 *}$ A. TSANG,${ }^{3}$ R. BUTLER, ${ }^{2}$ D. DAWSON,${ }^{4}$ D. GROOTHUIS,${ }^{5}$ \\ W. GROSS, ${ }^{6}$ J. HAWKINS, ${ }^{6}{ }^{\text {J. KILBURN }},{ }^{2}$ M. KUBIN,${ }^{7}$ K. H. SCHRÖDER,${ }^{8}$ V. A. SILCOX, ${ }^{2}$ \\ C. SMITH, ${ }^{2}$ M.-F. THOREL, ${ }^{9}$ C. WOODLEY,${ }^{2}$ AND M. A. YAKRUS ${ }^{2}$
}

Department of Veterans Affairs Medical Center, Long Beach, California $90822^{1}$; Centers for Disease Control, Atlanta, Georgia 303332; National Jewish Center for Immunology and Respiratory Disease, Denver, Colorado 80206 ${ }^{3}$; State Health Laboratory, Brisbane, Australia ; National Institute of Public Health, Bilthoven, The Netherlands ${ }^{5}$; Department of Veterans Affairs Medical Center, West Haven, Connecticut $06516^{6}$; National Institute of Public Health, Prague, Czechoslovakia ; Tuberkulose Forschungsinstitut, Borstel, Germany ; and Centre National d'Etudes Vétérinaires et Alimentaires, Laboratoire Central de Recherches Vétérinaires-BP 67, 94703 Maisons-Alfort Cedex, France ${ }^{9}$

\begin{abstract}
A cooperative study was conducted by the International Working Group on Mycobacterial Taxonomy to correlate the agglutination serovar designations of Mycobacterium avium, Mycobacterium intracellulare, and Mycobacterium scrofulaceum strains with the species ascriptions of these organisms according to molecular criteria and cultural properties and to assess the reproducibility of serovar determinations for a set of 63 reference strains of these species. Among the molecular criteria, the level of agreement between results obtained with nucleic acid probes and T-catalase serology results was $94 \%$ for strains of $M$. avium and $M$. intracellulare. Nucleic acid probes were not available for $M$. scrofulaceum, but none of the 10 strains ascribed to this species on the basis of catalase serology data reacted with a nucleic acid probe for $M$. avium or $M$. intracellulare. Ascription to a species on the basis of mycolic acid high-performance liquid chromatography patterns was in agreement with catalase serology results in $86 \%$ of the cases examined. Most strains belonging to serovars 1 through 6 and 8 through 11 were identified by molecular criteria as $M$. avium, most strains belonging to serovars 7,12 through 20,23 , and 25 were identified as $M$. intracellulare, and most strains belonging to serovars 41 through 43 were identified as $M$. scrofulaceum, in agreement with common current practice. Evidence for assigning serovar 27 to $M$. scrofulaceum was obtained. However, two strains of a given serovar may, on occasion, be placed in different species. The dominant species assignments for strains belonging to serovars 21, 24, 26, and 28 remain unresolved. Data from laboratories which used panels of sera that corresponded to all of the serovars represented in the study were in agreement with the consensus results in $84 \%$ of the instances. Laboratories in which a limited panel of sera was used were far more likely to report results that did not agree with the consensus results.
\end{abstract}

The dominant focus of most of the previous cooperative studies of the International Working Group on Mycobacterial Taxonomy (IWGMT) has been on the numerical taxonomy of the genus Mycobacterium (8, 10, 12, 14, 18, 21, 24-26), although some studies have been directed toward evaluation of the actual laboratory methods employed in mycobacterial systematics $(22,23)$. Systematic methods can fall into two categories. One category is based on physiologic or biochemical responses to a number of generally available chemical agents, and the tests can be performed independently in any laboratory. The other category is based on interaction with reference materials derived directly or indirectly from standard organisms; this type of method is specific to the group under study and requires the availability of reference materials, such as antibodies or nucleic acid probes. The previously published methodologic reports of the IWGMT addressed the first of these categories and included information about pigmentation, biochemical reactions, and resistance to inhibitors $(22,23)$. The second

\footnotetext{
* Corresponding author.
}

category of tests includes seroagglutination, which provides important infrasubspecific information on strains of such species as Mycobacterium avium, Mycobacterium intracellulare, and Mycobacterium scrofulaceum. The members of these three species produce similar polar glycopeptidolipid surface antigens that are responsible for very specific seroagglutination of whole cells (2). Standardized antisera are presently not generally available for agglutination typing of these organisms; those wishing to perform the tests must select individual strains that are representative of more than 30 serovars and use them to immunize rabbits. This can lead to interlaboratory discrepancies in the characterization of strains. The study reported here was designed to help evaluate the strains of $M$. avium, $M$. intracellulare, and $M$. scrofulaceum that serve as reference standards for each of the known agglutinating serovars and the comparability of results from laboratories in which different sera or minor modifications of techniques were used. Another purpose was to further test the species distributions of the infrasubspecific serovars with molecular reference standards, such as nucleic acid and antibody probes and high-performance liquid chromatography (HPLC) patterns of mycolic acids. 


\section{MATERIALS AND METHODS}

Strains. Serovars 1 through 28 , usually considered to belong in either $M$. avium or $M$. intracellulare, and serovars 41 through 43 , usually considered to belong in $M$. scrofulaceum, were represented in this study. A total of 63 strains from the reference collection of the National Jewish Center for Immunology and Respiratory Disease, Denver, Colo., were coded and distributed as unknown strains to all participants. As in all IWGMT studies, the code was not broken until the test results had been returned to the coordinator, and no new results were added to the data base once the code had been broken.

Tests. Workers in seven of the laboratories performed seroagglutination tests by using methods pioneered by Schaefer (16), with minor individual modifications as noted below. Unless otherwise noted, each laboratory produced its own rabbit antisera to strains selected as representative for each of the serovars. Enzyme-linked immunosorbent assays (ELISA) and chromatographic analyses were also performed in one laboratory to detect the individual glycopeptidolipid antigens responsible for specific seroagglutination. In addition to the testing of cells for serovar identification, extracts of the strains were subjected to DNA-RNA probe (GenProbe, Inc., San Diego, Calif.) (15) and catalase antibody probe $(20)$ analyses, and their HPLC patterns were determined (4), to help assign the strains to their correct species.

(i) Laboratory 1 . In laboratory 1 the strains were typed by the tube agglutination method described by Good and Beam (7). Sera against serovars 1 through 28 and 41 through 43 were used. Unless otherwise noted, agglutination reactions were confirmed by cross-absorption tests.

(ii) Laboratory 2. In laboratory 2 only sera against serovars 1 through 11 were used. The sera were not crossabsorbed, and the method of Schaefer was used (16).

(iii) Laboratory 3. In laboratory 3 strains were tested with sera against serovars 1 through 28 and 41 through 43 by using the tube agglutination method of Schaefer (16). The serum designated Cole by workers in this laboratory corresponds to the serum of previously proposed $M$. scrofulaceum serovar 44 (9), which was later recognized to be synonymous with serovar $27(30)$, and the designation serovar 27 is used in this report. Serovars 41,42 , and 43 were reported as serovars scrofulaceum, Lunning, and Gause, respectively, according to the convention that preceded the current numerical coding of serovars (30) and were translated to current code designations by the coordinator.

(iv) Laboratory 4. In laboratory 4 a slide agglutination technique was used; only sera against serovars 1 through 20 were used.

(v) Laboratory 5. In laboratory 5 strains were tested against serovars 1 through 28 and 41 through 43 by using a microtube agglutination test (13).

(vi) Laboratory 6. In laboratory 6 only sera against serovars $1,2,4$ through 11,13 through 23,25 through 27 , and 41 were used, and agglutination tests were performed by using the microtube modification (13). The sera were not produced in this laboratory, but were obtained from the laboratory of the National Jewish Center for Immunology and Respiratory Disease. Serovar 41 was reported as serovar scrofulaceum, according to the convention that preceded the current numerical coding of serovars (30).

(vii) Laboratory 7. Workers in laboratory 7 assumed the responsibility for assembling, coding, and distributing the 63 strains to be tested. The composite results of seroagglutination tests, an ELISA performed with murine monoclonal and rabbit polyclonal antibodies, thin-layer chromatography tests, and, in some cases, gas chromatography tests $(5,17)$ were used by workers in this laboratory to confirm the concordance of the data obtained with the original strain serovar designations.

(viii) Laboratory 8. In laboratory $8 \mathrm{~T}$-catalase intrinsic enzyme dot blot serologic testing (20) was performed with sonic extracts of the test strains and cross-absorbed sera specific for four species $(M$. avium, $M$. intracellulare, $M$. scrofulaceum, and Mycobacterium tuberculosis), as well as a dot of nonabsorbed polyvalent antibody to confirm the presence of T-catalase in each extract.

(ix) Laboratory 9. In laboratory 9 sonic extracts of all strains were examined with DNA probes specific for rRNAs of $M$. avium and $M$. intracellulare (Gen-Probe).

(x) Laboratory 10 . In laboratory 10 extracts of all strains were examined by HPLC, and selected mycolic acid peaks were used to establish the identities of strains (4).

(xi) Laboratory 11. In laboratory 11 the cultures were examined for conventional phenotypic cultural properties, including pigment, Tween hydrolysis, catalase, growth temperature, nitrate reduction, and 3-day aryl sulfatase activity $(22,23)$.

\section{RESULTS}

The codes, original designations, and nominal serovars of all of the reference strains distributed for this study are listed in Table 1. Strain 6 failed to grow and was excluded from consideration.

The results of all tests except the tests for the cultural properties (i.e., pigmentation and physiologic and biochemical activities) are shown by strain and by laboratory in Table 2 . The sequence in which the strains are listed is based on the consensus serovar results but distributed among each of the species categories on the basis of the nucleic acid, catalase antibody, and HPLC data. The confirmatory result obtained by the distributing laboratory, laboratory 7 , is shown for each strain, along with the seroagglutination results from laboratories 1,3 , and 5 and the consensus serovar results. The consensus results represent agreement of the reports from two or more of the four laboratories that used a panel of sera that included all of the serovars in the study (laboratories $1,3,5$, and 7). The results from laboratories 2,4 , and 6 , which were based on an incomplete panel of sera, are also shown.

The cultural properties were in general agreement with the species assignments based on molecular analyses. With few exceptions, $M$. avium and $M$. intracellulare strains grew into buff to pale yellow colonies and generated less than $45 \mathrm{~mm}$ of foam in the semiquantitative catalase test; the strains identified as $M$. scrofulaceum were scotochromogenic, exhibiting a bright yellow to orange pigment, and generated $45 \mathrm{~mm}$ or more of foam in the catalase test. Of special interest is the observation that $15(75 \%)$ of the $M$. avium strains grew at $45^{\circ} \mathrm{C}$, whereas none of the strains identified as $M$. intracellulare or $M$. scrofulaceum were able to grow at that temperature. None of the strains in the study gave significant reactions in the nitrate reduction test. Data for strains which gave discrepant results in any of the tests or with which species crossover of serovars was seen are presented in Table 3.

As shown in Table 2, the Gen-Probe and T-catalase serologic data were in complete accord for the first 20 strains identified as $M$. avium and corresponded to serovars 1 through 6,8 through 11 , and 21 ; five of these 20 strains were 
TABLE 1. Source designations and nominal serovars of reference strains coded and distributed for this study

\begin{tabular}{|c|c|c|c|c|c|}
\hline Strain & $\begin{array}{c}\text { Source } \\
\text { designation }\end{array}$ & $\begin{array}{c}\text { Nominal } \\
\text { serovar }\end{array}$ & Strain & $\begin{array}{c}\text { Source } \\
\text { designation }\end{array}$ & $\begin{array}{c}\text { Nominal } \\
\text { serovar }\end{array}$ \\
\hline 1 & $11907-300$ & 1 & 33 & P-49 & 7 \\
\hline 2 & Melnick & 18 & 34 & Wood Duck & 12 \\
\hline 3 & P-39 & 14 & 35 & Harrison & 27 \\
\hline 4 & EW 10407 & 42 & 36 & $72-888$ & 25 \\
\hline 5 & 157 Manten & 7 & 37 & CDC 1217 & 23 \\
\hline 7 & 1195 CDC & 25 & 38 & 128 Germany & 3 \\
\hline 8 & W 552 & 19 & 39 & McKenzie 13723 & 26 \\
\hline 9 & $14816-1424$ & 11 & 40 & ATCC 15987 & 16 \\
\hline 10 & 23393 & 23 & 41 & TMC 1419 & 20 \\
\hline 11 & 2993 & 21 & 42 & 10409 & 22 \\
\hline 12 & TMC 1461 & 10 & 43 & 9055 Matthews & 28 \\
\hline 13 & 2729 Cardiff & 41 & 44 & Brooks & 43 \\
\hline 14 & Darden & 19 & 45 & TMC 1473 & 15 \\
\hline 15 & $4443-1237$ & 5 & 46 & M-150 & 43 \\
\hline 16 & 34540 & 6 & 47 & Edgar Boone & 14 \\
\hline 17 & B-92 & 1 & 48 & Yandle & 16 \\
\hline 18 & $6450-204$ & 9 & 49 & $1602-1965$ & 10 \\
\hline 19 & $14141-1395$ & 2 & 50 & Hillberry $1244-9$ & 26 \\
\hline 20 & 4990 O'Connor & 18 & 51 & 12645 & 24 \\
\hline 21 & ATCC 23435 & 8 & 52 & P-54 & 17 \\
\hline 22 & Bridge & 41 & 53 & Simpson & 15 \\
\hline 23 & $25546-759$ & 5 & 54 & TMC 1463 & 4 \\
\hline 24 & 6845 & 28 & 55 & 6194 & 2 \\
\hline 25 & AT 545 Findley & 20 & 56 & Leonard-158 & 7 \\
\hline 26 & 6195 & 3 & 57 & Anderson & 43 \\
\hline 27 & Lane 3081 & 27 & 58 & 5154 O'Connor & 22 \\
\hline 28 & $1784-286$ & 9 & 59 & P-42 & 12 \\
\hline 29 & SJB \#2 & 8 & 60 & 16741 Cardiff & 2 \\
\hline 30 & TMC 1466 & 13 & 61 & Lunning CDC 1198 & 42 \\
\hline 31 & TMC 1462 & 11 & 62 & $13528-1079$ & 4 \\
\hline 32 & ATCC 25122 & 13 & 63 & TMC 1406 & 16 \\
\hline
\end{tabular}

identified as $M$. intracellulare on the basis of the HPLC patterns, but the interpretation of HPLC curves at the time that this study was done was based on restricted peak comparisons (4). Recent computer-based programs for pattern recognition have led to more accurate identification by HPLC (3). Typical phenotypic culture patterns were observed for the $M$. avium strains, with a few exceptions; three strains gave catalase reactions which generated more than $\mathbf{4 5}$ $\mathrm{mm}$ of foam (Table 3 ), and one strain was reported to give a positive reaction in the Tween hydrolysis test.

Only three discrepancies were observed between the Gen-Probe nucleic acid and the T-catalase serologic results among the next 28 strains in Table 2; these 28 strains were identified as $M$. intracellulare and included all available members of serovars 12 through 20,23 , and 25 , as well as one or more members of serovars 7,26 , and 43 . Of special interest is the group of three strains belonging to serovar 23, which reacted like $M$. intracellulare with the T-catalase probe but failed to react or reacted like $M$. avium with the DNA probe. This group is discussed further below along with the molecular nonconsensus strains listed in Table 2 . A total of 26 of the strains in the $M$. intracellulare group were identified as members of that species by HPLC; strains 7 and 36 , both serovar 25 strains, were reported to be a mixed culture or to have the $M$. scrofulaceum HPLC pattern, respectively. Three of the strains in the $M$. intracellulare group differed in one or more cultural properties from the typical pattern for this species; one strain produced orange pigment but had low catalase activity, one generated more than $45 \mathrm{~mm}$ of foam in the semiquantitative catalase test but was not pigmented, and the third produced a typical $M$. scrofulaceum phenotypic pattern of scotochromogenicity and high catalase activity (Table 3 ).

Ten strains were recognized as $M$. scrofulaceum on the basis of both T-catalase serologic test and HPLC patterns. There is no Gen-Probe kit for this species; thus, all 10 of these strains were correctly recorded as not identifiable with the available DNA probes. Six of the seven strains belonging to traditional (30) scrofulaceum serovars 41 through 43 were identified as $M$. scrofulaceum; one serovar 43 strain (strain 57) was identified by molecular methods and on the basis of cultural phenotypic properties as $M$. intracellulare (Table 2). One serovar 7 strain (strain 33) was identified by molecular and cultural methods as $M$. scrofulaceum, in contrast to two other strains of this serovar that were identified as $M$. intracellulare, as expected.

As noted previously, Goslee and colleagues (9) proposed a fourth serovar in the $M$. scrofulaceum group and designated it serovar 44. Wolinsky later recognized this serovar to be synonymous with "the recently established serovar 27 of the M. avium complex," citing a communication from D. Dawson, and withdrew the proposal of serovar 44 (29). We found no published phenotypic description of the original strains attributed to serovar 27, but one of us (D.D.) advises that the original representatives of both serovars 26 and 27 exhibited phenotypic properties characteristic of $M$. scrofulaceum and that neither was ever designated a member of the $M$. avium complex. Despite this, Schaefer assigned series 20 numerical designations, which by convention are reserved for the $M$. avium complex, to these two serovars instead of numbers in series 40 , which are used for serovars of $M$. scrofulaceum (7). Both of the strains recognized as serovar 27 strains and 
TABLE 2. Species as determined by molecular methods and serovars as determined by agglutination of 62 coded strains of $M$. avium, $M$. intracellulare, $M$. scrofulaceum, and related mycobacteria

\begin{tabular}{|c|c|c|c|c|c|c|c|c|c|c|c|c|}
\hline \multirow{2}{*}{ Strain } & \multicolumn{4}{|c|}{$\begin{array}{l}\text { Species identification as determined by } \\
\text { molecular methods }\end{array}$} & \multicolumn{8}{|c|}{ Serovar(s) as determined by agglutination } \\
\hline & Catalase & Gen-Probe & HPLC & Consensus & $\begin{array}{l}\text { Labora- } \\
\text { tory } 7\end{array}$ & $\begin{array}{l}\text { Labora- } \\
\text { tory } 1\end{array}$ & $\begin{array}{l}\text { Labora- } \\
\text { tory } 3\end{array}$ & $\begin{array}{l}\text { Labora- } \\
\text { tory } 5\end{array}$ & $\begin{array}{l}\text { Consen- } \\
\text { sus }^{a}\end{array}$ & $\begin{array}{l}\text { Labora- } \\
\text { tory } 2\end{array}$ & $\begin{array}{l}\text { Labora- } \\
\text { tory } 4\end{array}$ & $\begin{array}{l}\text { Labora- } \\
\text { tory } 6\end{array}$ \\
\hline 1 & M. avium & M. avium & M. avium & M. avium & 1 & $\mathrm{SA}^{b}$ & $\mathrm{NRX}^{c}$ & $1 ?, \mathrm{SA}$ & $\mathrm{NC}^{d}$ & $1(2,3)^{e}$ & 1 & 1 \\
\hline 17 & M. avium & M. avium & $\begin{array}{l}\text { M. intracel- } \\
\text { lulare }\end{array}$ & M. avium & 1 & $1^{f}$ & 17 & SA & 1 & 1 & 3 & SA \\
\hline 19 & M. avium & M. avium & M. avium & M. avium & 2 & 2 & 2 & $2 ?$ & 2 & 2 & 3 & SA \\
\hline 55 & M. avium & M. avium & $\begin{array}{l}\text { M. intracel- } \\
\text { lulare }\end{array}$ & M. avium & 2 & $1^{f}$ & 24,25 & 2 & 2 & SA & SA & 8 \\
\hline 60 & M. avium & M. avium & M. avium & M. avium & 2 & 2 & NRX & $2 ?$ & 2 & NRX & 3 & SA \\
\hline 26 & M. avium & M. avium & $\begin{array}{l}\text { M. intracel- } \\
\text { lulare }\end{array}$ & M. avium & $\mathrm{NT}^{\mathrm{g}}$ & 3 & 3,2 & 3 & 3 & 2 & 3 & NT \\
\hline 38 & M. avium & M. avium & M. avium & M. avium & $3,23,25$ & 3 & SA & 3 ? & 3 & 7 & SA & NRX \\
\hline 54 & M. avium & M. avium & M. avium & M. avium & 4 & 4 & 4 & 4 & 4 & NRX & 4 & 19 \\
\hline 62 & M. avium & M. avium & M. avium & M. avium & 4 & 4 & 4 & 4 & 4 & SA & 4 & NRX \\
\hline 15 & M. avium & M. avium & $\begin{array}{l}\text { M. intracel- } \\
\text { lulare }\end{array}$ & M. avium & 5 & 5 & 5 & NRX & 5 & NRX & 5 & 4 \\
\hline 23 & M. avium & M. avium & $M$. avium & M. avium & 5 & 5 & 5 & NRX & 5 & NRX & 5 & SA \\
\hline 16 & $M$, avium & M. avium & M. avium & M. avium & 6,8 & SA & 6 & SA & 6 & $8(2,3,6)$ & 6 & 6 \\
\hline 29 & M. avium & M. avium & M. avium & M. avium & 8 & 8 & 8 & 8 & 8 & 8 & 8 & NRX \\
\hline 18 & M. avium & M. avium & $\begin{array}{l}\text { M. intracel- } \\
\text { lulare }\end{array}$ & M. avium & 9 & 9 & 9 & 9 & 9 & 10 & 9 & NRX \\
\hline 28 & M. avium & M. avium & M. avium & M. avium & 9 & 9 & 9 & $9 ?$ & 9 & SA & 9 & SA \\
\hline 12 & M. avium & M. avium & M. avium & M. avium & 10 & 10 & 10 & 10 & 10 & 8,1 & 10 & NRX \\
\hline 49 & M. avium & M. avium & M. avium & M. avium & 10 & 10 & 10 & 10 & 10 & NRX & 4 & NRX \\
\hline 9 & M. avium & M. avium & M. avium & $M$. avium & 11 & 11,10 & 11,10 & 11 & 11 & NRX & 11 & SA \\
\hline 31 & M. avium & M. avium & M. avium & M. avium & 11 & SA & 11,10 & 11 & 11 & NRX & 11 & NT \\
\hline 11 & $M$, avium & M. avium & M. avium & M. avium & 21 & 21 & 21,8 & 21,8 & 21 & 8,1 & NRX & 4 \\
\hline 5 & $\begin{array}{l}\text { M. intracel- } \\
\text { lulare }\end{array}$ & $\begin{array}{l}\text { M. intracel- } \\
\text { lulare }\end{array}$ & $\begin{array}{l}\text { M. intracel- } \\
\text { lulare }\end{array}$ & $\begin{array}{l}\text { M. intracel- } \\
\text { lulare }\end{array}$ & 7 & $7,13^{f}$ & 7 & 7 & 7 & NRX & 7 & 7 \\
\hline 56 & $\begin{array}{l}\text { M. intracel- } \\
\text { lulare }\end{array}$ & $\begin{array}{l}\text { M. intracel- } \\
\text { lulare }\end{array}$ & $\begin{array}{l}\text { M. intracel- } \\
\text { lulare }\end{array}$ & $\begin{array}{l}\text { M. intracel- } \\
\text { lulare }\end{array}$ & 7 & 7 & 7,13 & 7 & 7 & 7 & 7 & NRX \\
\hline 24 & $\begin{array}{l}\text { M. intracel- } \\
\text { lulare }\end{array}$ & $\begin{array}{l}M . \text { intracel- } \\
\text { lulare }\end{array}$ & $\begin{array}{l}\text { M. intracel- } \\
\text { lulare }\end{array}$ & $\begin{array}{l}\text { M. intracel- } \\
\text { lulare }\end{array}$ & 12 & 12,28 & 12 & 12 & 12 & 1,2 & 12 & NRX \\
\hline 34 & $\begin{array}{l}\text { M. intracel- } \\
\text { lulare }\end{array}$ & $\begin{array}{l}\text { M. intracel- } \\
\text { lulare }\end{array}$ & $\begin{array}{l}\text { M. intracel- } \\
\text { lulare }\end{array}$ & $\begin{array}{l}\text { M. intracel- } \\
\text { lulare }\end{array}$ & 12 & 12 & 7,13 & 12 & 12 & NRX & 12 & NRX \\
\hline 59 & $\begin{array}{l}\text { M. intracel- } \\
\text { lulare }\end{array}$ & $\begin{array}{l}\text { M. intracel- } \\
\text { lulare }\end{array}$ & $\begin{array}{l}\text { M. intracel- } \\
\text { lulare }\end{array}$ & $\begin{array}{l}\text { M. intracel- } \\
\text { lulare }\end{array}$ & 12 & 12,28 & 12 & 12 & 12 & (7) & 12,13 & NRX \\
\hline 30 & $\begin{array}{l}\text { M. intracel- } \\
\text { lulare }\end{array}$ & $\begin{array}{l}\text { M. intracel- } \\
\text { lulare }\end{array}$ & $\begin{array}{l}\text { M. intracel- } \\
\text { lulare }\end{array}$ & $\begin{array}{l}\text { M. intracel- } \\
\text { lulare }\end{array}$ & 13 & 13 & 13 & 13 & 13 & 7 & 13 & SA \\
\hline 32 & $\begin{array}{l}\text { M. intracel- } \\
\text { lulare }\end{array}$ & $\begin{array}{l}\text { M. intracel- } \\
\text { lulare }\end{array}$ & $\begin{array}{l}\text { M. intracel- } \\
\text { lulare }\end{array}$ & $\begin{array}{l}\text { M. intracel- } \\
\text { lulare }\end{array}$ & 13 & 13 & 13 & 13 & 13 & 7 & 12 & 13 \\
\hline 3 & $\begin{array}{l}\text { M. intracel- } \\
\text { lulare }\end{array}$ & $\begin{array}{l}\text { M. intracel- } \\
\text { lulare }\end{array}$ & $\begin{array}{l}\text { M. intracel- } \\
\text { lulare }\end{array}$ & $\begin{array}{l}\text { M. intracel- } \\
\text { lulare }\end{array}$ & 14 & SA & 14 & 14 & 14 & 11 & 14 & NRX \\
\hline 47 & $\begin{array}{l}\text { M. intracel- } \\
\text { lulare }\end{array}$ & $\begin{array}{l}\text { M. intracel- } \\
\text { lulare }\end{array}$ & $\begin{array}{l}\text { M. intracel- } \\
\text { lulare }\end{array}$ & $\begin{array}{l}\text { M. intracel- } \\
\text { lulare }\end{array}$ & 14 & 14 & NRX & 14 & 14 & 11 & 14 & 14 \\
\hline 21 & $\begin{array}{l}\text { M. intracel- } \\
\text { lulare }\end{array}$ & $\begin{array}{l}\text { M. intracel- } \\
\text { lulare }\end{array}$ & $\begin{array}{l}\text { M. intracel- } \\
\text { lulare }\end{array}$ & $\begin{array}{l}\text { M. intracel- } \\
\text { lulare }\end{array}$ & 15 & 15,42 & 19 & 15 & 15 & NRX & 15 & NRX \\
\hline 45 & $\begin{array}{l}M . \text { intracel- } \\
\text { lulare }\end{array}$ & $\begin{array}{l}\text { M. intracel- } \\
\text { lulare }\end{array}$ & $\begin{array}{l}\text { M. intracel- } \\
\text { lulare }\end{array}$ & $\begin{array}{l}\text { M. intracel- } \\
\text { lulare }\end{array}$ & 15 & 15,42 & NRX & 15 & 15 & SA & 15 & 9 \\
\hline 53 & $\begin{array}{l}\text { M. intracel- } \\
\text { lulare }\end{array}$ & $\begin{array}{l}\text { M. intracel- } \\
\text { lulare }\end{array}$ & $\begin{array}{l}\text { M. intracel- } \\
\text { lulare }\end{array}$ & $\begin{array}{l}\text { M. intracel- } \\
\text { lulare }\end{array}$ & 15 & 15,42 & NRX & 15 & 15 & SA & 15 & NRX \\
\hline 40 & $\begin{array}{l}\text { M. intracel- } \\
\text { lulare }\end{array}$ & $\begin{array}{l}\text { M. intracel- } \\
\text { lulare }\end{array}$ & $\begin{array}{l}\text { M. intracel- } \\
\text { lulare }\end{array}$ & $\begin{array}{l}\text { M. intracel- } \\
\text { lulare }\end{array}$ & 16 & 16 & 16 & 16 & 16 & NRX & 16 & NRX \\
\hline 48 & $\begin{array}{l}\text { M. intracel- } \\
\text { lulare }\end{array}$ & $\begin{array}{l}\text { M. intracel- } \\
\text { lulare }\end{array}$ & $\begin{array}{l}\text { M. intracel- } \\
\text { lulare }\end{array}$ & $\begin{array}{l}\text { M. intracel- } \\
\text { lulare }\end{array}$ & 16 & 16 & 16 & 16 & 16 & NRX & 16 & NRX \\
\hline 63 & $\begin{array}{l}\text { M. intracel- } \\
\text { lulare }\end{array}$ & $\begin{array}{l}M . \text { intracel- } \\
\text { lulare }\end{array}$ & $\begin{array}{l}\text { M. intracel- } \\
\text { lulare }\end{array}$ & $\begin{array}{l}\text { M. intracel- } \\
\text { lulare }\end{array}$ & 16 & 16 & 16 & 16 & 16 & $6,7,8,11$ & 16 & NRX \\
\hline 52 & $\begin{array}{l}\text { M. intracel- } \\
\text { lulare }\end{array}$ & $\begin{array}{l}\text { M. intracel- } \\
\text { lulare }\end{array}$ & $\begin{array}{l}\text { M. intracel- } \\
\text { lulare }\end{array}$ & $\begin{array}{l}\text { M. intracel- } \\
\text { lulare }\end{array}$ & 17 & 17 & 17 & 17 & 17 & (7) & 17 & 16 \\
\hline 20 & $\begin{array}{l}\text { M. intracel- } \\
\text { lulare }\end{array}$ & $\begin{array}{l}\text { M. intracel- } \\
\text { lulare }\end{array}$ & $\begin{array}{l}\text { M. intracel- } \\
\text { lulare }\end{array}$ & $\begin{array}{l}\text { M. intracel- } \\
\text { lulare }\end{array}$ & 9 & 18 & 18 & 18 & 18 & NRX & 18 & 2 \\
\hline 8 & $\begin{array}{l}\text { M. intracel- } \\
\text { lulare }\end{array}$ & $\begin{array}{l}\text { M. intracel- } \\
\text { lulare }\end{array}$ & $\begin{array}{l}\text { M. intracel- } \\
\text { lulare }\end{array}$ & $\begin{array}{l}\text { M. intracel- } \\
\text { lulare }\end{array}$ & 19 & 19 & 19 & $19 ?$ & 19 & NRX & NRX & NRX \\
\hline 14 & $\begin{array}{l}\text { M. intracel- } \\
\text { lulare }\end{array}$ & $\begin{array}{l}\text { M. intracel- } \\
\text { lulare }\end{array}$ & $\begin{array}{l}\text { M. intracel- } \\
\text { lulare }\end{array}$ & $\begin{array}{l}\text { M. intracel- } \\
\text { lulare }\end{array}$ & 19 & 19 & 19 & 19 & 19 & $1,2(11)$ & 1 & 19 \\
\hline
\end{tabular}


TABLE 2-Continued

\begin{tabular}{|c|c|c|c|c|c|c|c|c|c|c|c|c|}
\hline \multirow{2}{*}{ Strain } & \multicolumn{4}{|c|}{$\begin{array}{l}\text { Species identification as determined by } \\
\text { molecular methods }\end{array}$} & \multicolumn{8}{|c|}{ Serovar(s) as determined by agglutination } \\
\hline & Catalase & Gen-Probe & HPLC & Consensus & $\begin{array}{l}\text { Labora- } \\
\text { tory } 7\end{array}$ & $\begin{array}{l}\text { Labora- } \\
\text { tory } 1\end{array}$ & $\begin{array}{l}\text { Labora- } \\
\text { tory } 3\end{array}$ & $\begin{array}{l}\text { Labora- } \\
\text { tory } 5\end{array}$ & $\begin{array}{l}\text { Consen- } \\
\text { sus }^{a}\end{array}$ & $\begin{array}{l}\text { Labora- } \\
\text { tory } 2\end{array}$ & $\begin{array}{l}\text { Labora- } \\
\text { tory } 4\end{array}$ & $\begin{array}{l}\text { Labora- } \\
\text { tory } 6\end{array}$ \\
\hline 41 & $\begin{array}{l}\text { M. intracel- } \\
\text { lulare }\end{array}$ & $\begin{array}{l}\text { M. intracel- } \\
\text { lulare }\end{array}$ & $\begin{array}{l}\text { M. intracel- } \\
\text { lulare }\end{array}$ & $\begin{array}{l}\text { M. intracel- } \\
\text { lulare }\end{array}$ & 20 & $1^{f}$ & 20 & NT & 20 & NT & SA & SA \\
\hline 25 & $\begin{array}{l}\text { M. intracel- } \\
\text { lulare }\end{array}$ & $\begin{array}{l}\text { M. intracel- } \\
\text { lulare }\end{array}$ & $\begin{array}{l}\text { M. intracel- } \\
\text { lulare }\end{array}$ & $\begin{array}{l}\text { M. intracel- } \\
\text { lulare }\end{array}$ & 21 & 20 & 14 & NRX & $\mathrm{NC}$ & $11(5)$ & SA & 5 \\
\hline 10 & $\begin{array}{l}\text { M. intracel- } \\
\text { lulare }\end{array}$ & M. avium & $\begin{array}{l}\text { M. intracel- } \\
\text { lulare }\end{array}$ & $\begin{array}{l}\text { M. intracel- } \\
\text { lulare }\end{array}$ & 23 & 23 & 9 & 23 & 23 & 8,1 & 7 & 23 \\
\hline 37 & $\begin{array}{l}\text { M. intracel- } \\
\text { lulare }\end{array}$ & NRX & $\begin{array}{l}\text { M. intracel- } \\
\text { lulare }\end{array}$ & $\begin{array}{l}\text { M. intracel- } \\
\text { lulare }\end{array}$ & 23 & 23 & 23 & 23 & 23 & 7 & 7 & 23 \\
\hline 51 & $\begin{array}{l}\text { M. intracel- } \\
\text { lulare }\end{array}$ & NRX & $\begin{array}{l}\text { M. intracel- } \\
\text { lulare }\end{array}$ & $\begin{array}{l}\text { M. intracel- } \\
\text { lulare }\end{array}$ & 23 & 23,24 & 23 & 23 & 23 & 7 & 7 & 23 \\
\hline 7 & $\begin{array}{l}M \text {. intracel- } \\
\text { lulare }\end{array}$ & $\begin{array}{l}\text { M. intracel- } \\
\text { lulare }\end{array}$ & $\begin{array}{l}\text { Mixed cul- } \\
\text { ture? }\end{array}$ & $\begin{array}{l}\text { M. intracel- } \\
\text { lulare }\end{array}$ & 25 & 25 & SA & 25 & 25 & 1,2 & 15 & SA \\
\hline 36 & $\begin{array}{l}\text { M. intracel- } \\
\text { lulare }\end{array}$ & $\begin{array}{l}\text { M. intracel- } \\
\text { lulare }\end{array}$ & $\begin{array}{l}\text { M. scrofu- } \\
\text { laceum }\end{array}$ & $\begin{array}{l}M . \text { intracel- } \\
\text { lulare }\end{array}$ & 25 & 25 & NRX & 25 & 25 & $7(8)$ & SA & SA \\
\hline 50 & $\begin{array}{l}\text { M. intracel- } \\
\text { lulare }\end{array}$ & $\begin{array}{l}\text { M. intracel- } \\
\text { lulare }\end{array}$ & $\begin{array}{l}\text { M. intracel- } \\
\text { lulare }\end{array}$ & $\begin{array}{l}\text { M. intracel- } \\
\text { lulare }\end{array}$ & 26 & 26 & 26 & 26 & 26 & NRX & SA & NRX \\
\hline 57 & $\begin{array}{l}\text { M. intracel- } \\
\text { lulare }\end{array}$ & $\begin{array}{l}\text { M. intracel- } \\
\text { lulare }\end{array}$ & $\begin{array}{l}\text { M. intracel- } \\
\text { lulare }\end{array}$ & $\begin{array}{l}\text { M. intracel- } \\
\text { lulare }\end{array}$ & 43 & 43 & NRX & 43 & 43 & 1 & NRX & NRX \\
\hline 33 & $\begin{array}{l}\text { M. scrofu- } \\
\text { laceum }\end{array}$ & NRX & $\begin{array}{c}\text { M. scrofu- } \\
\text { laceum }\end{array}$ & $\begin{array}{c}\text { M. scrofu- } \\
\text { laceum }\end{array}$ & 7 & $\begin{array}{l}7,1,12 \\
13\end{array}$ & 12 & 7 & 7 & 7 & 7 & 7 \\
\hline 39 & $\begin{array}{l}\text { M. scrofu- } \\
\text { laceum }\end{array}$ & NRX & $\begin{array}{l}\text { M. scrofu- } \\
\text { laceum }\end{array}$ & $\begin{array}{l}\text { M. scrofu- } \\
\text { laceum }\end{array}$ & 26 & 26 & 26 & 26 & 26 & NRX & NRX & 26 \\
\hline 27 & $\begin{array}{l}\text { M. scrofu- } \\
\text { laceum }\end{array}$ & NRX & $\begin{array}{l}\text { M. scrofu- } \\
\text { laceum }\end{array}$ & $\begin{array}{l}\text { M. scrofu- } \\
\text { laceum }\end{array}$ & 27 & NRX & 27 & 27 & 27 & NRX & NRX & NRX \\
\hline 35 & $\begin{array}{l}\text { M. scrofu- } \\
\text { laceum }\end{array}$ & NRX & $\begin{array}{l}\text { M. scrofu- } \\
\text { laceum }\end{array}$ & $\begin{array}{l}\text { M. scrofu- } \\
\text { laceum }\end{array}$ & 27 & $27^{f}$ & 27 & 27 & 27 & NRX & NRX & 27 \\
\hline 13 & $\begin{array}{l}\text { M. scrofu- } \\
\text { laceum }\end{array}$ & NRX & $\begin{array}{l}\text { M. scrofu- } \\
\text { laceum }\end{array}$ & $\begin{array}{l}\text { M. scrofu- } \\
\text { laceum }\end{array}$ & 41 & 41 & $20,24,25$ & 41 & 41 & NRX & 20 & 41 \\
\hline 22 & $\begin{array}{l}\text { M. scrofu- } \\
\text { laceum }\end{array}$ & NRX & $\begin{array}{l}\text { M. scrofu- } \\
\text { laceum }\end{array}$ & $\begin{array}{l}\text { M. scrofu- } \\
\text { laceum }\end{array}$ & 41 & 41 & 8 & 41 & 41 & NRX & 20 & 41 \\
\hline 4 & $\begin{array}{l}\text { M. scrofu- } \\
\text { laceum }\end{array}$ & NRX & $\begin{array}{l}\text { M. scrofu- } \\
\text { laceum }\end{array}$ & $\begin{array}{l}\text { M. scrofu- } \\
\text { laceum }\end{array}$ & 42 & $42,1^{f}$ & 42 & 42 & 42 & NRX & NRX & SA \\
\hline 61 & $\begin{array}{l}\text { M. scrofu- } \\
\text { laceum }\end{array}$ & NRX & $\begin{array}{l}\text { M. intracel- } \\
\text { lulare }\end{array}$ & $\begin{array}{l}\text { M. scrofu- } \\
\text { laceum }\end{array}$ & 42 & $1^{f}$ & NRX & 42 & 42 & NRX & SA & NRX \\
\hline 44 & $\begin{array}{l}\text { M. scrofu- } \\
\text { laceum }\end{array}$ & NRX & $\begin{array}{l}\text { M. scrofu- } \\
\text { laceum }\end{array}$ & $\begin{array}{l}\text { M. scrofu- } \\
\text { laceum }\end{array}$ & 43 & 43 & 43 & 43 & 43 & NRX & NRX & 8 \\
\hline 46 & $\begin{array}{l}\text { M. scrofu- } \\
\text { laceum }\end{array}$ & NRX & $\begin{array}{l}\text { M. scrofu- } \\
\text { laceum }\end{array}$ & $\begin{array}{l}\text { M. scrofu- } \\
\text { laceum }\end{array}$ & 43 & 43 & 43 & 43 & 43 & NRX & NRX & 8 \\
\hline 2 & NT & NRX & $\begin{array}{l}\text { M. intracel- } \\
\text { lulare }\end{array}$ & $\mathrm{NC}$ & 18 & 18 & 18 & 18 & 18 & NT & 18 & SA \\
\hline 42 & $\begin{array}{l}\text { M. scrofu- } \\
\text { laceum }\end{array}$ & NRX & M. avium & $\mathrm{NC}$ & 22 & 22 & 22 & 22 & 22 & 7 & 7 & 13 \\
\hline 58 & $\begin{array}{l}\text { M. intracel- } \\
\text { lulare }\end{array}$ & NRX & $?$ & $\mathrm{NC}$ & 22 & 22 & 22 & 22 & 22 & NRX & 7 & 22 \\
\hline 43 & $\begin{array}{l}\text { M. intracel } \text { - } \\
\text { lulare }\end{array}$ & NRX & $\begin{array}{l}\text { M. scrofu- } \\
\text { laceum }\end{array}$ & $\mathrm{NC}$ & 28 & 28 & 28,24 & 28 & 23 & NT & NRX & 2 \\
\hline
\end{tabular}

a Consensus serovar results are based on the data from laboratories $1,3,5$, and 7 .

${ }^{b} \mathrm{SA}$, spontaneous agglutination.

$c$ NRX, no reaction with any of the reference antisera or probes tested.

${ }^{d} \mathrm{NC}$, no consensus.

e Parentheses indicate weak reactions $(\leq 2+)$.

$f$ Not confirmed by cross-absorption.

$g$ NT, test not performed.

one of the two strains recognized as serovar 26 strains in this study were characterized as $M$. scrofulaceum. It is also of interest that Saito and colleagues previously examined the two source strains of serovars 26 and 27 with cross-absorbed antibody to their alpha antigens and reported that all four strains react as $M$. scrofulaceum strains (15).

For four strains (strains 2, 42, 43, and 58), no consensus on ascription to a species on the basis of molecular data could be reached. In addition, two strains (strains 37 and 51) were assigned to $M$. intracellulare on the basis of T-catalase serology and HPLC results, but they could not be identified by the Gen-Probe kits. Similar problems were encountered in ascribing members of an $M$. avium complex-like cluster of strains (cluster 4) to a species in a recent IWGMT numerical taxonomic report (27).

When the observed serovar assignments were compared 
TABLE 3. Characteristics of strains whose identification is ambiguous

\begin{tabular}{|c|c|c|c|c|c|c|c|c|}
\hline \multirow{2}{*}{ Strain } & \multicolumn{3}{|c|}{ Species as determined by molecular methods } & \multirow{2}{*}{$\begin{array}{l}\text { Consensus } \\
\text { serovar }\end{array}$} & \multirow{2}{*}{ Pigment } & \multirow{2}{*}{$\begin{array}{c}\text { Catalase } \\
\text { reaction } \\
\text { (mm of } \\
\text { foam) }\end{array}$} & \multirow{2}{*}{$\begin{array}{l}\text { Growth } \\
\text { at } 45^{\circ} \mathrm{C}^{a}\end{array}$} & \multirow{2}{*}{$\begin{array}{c}\text { Tween } \\
\text { hydrolysis }\end{array}$} \\
\hline & Catalase & Gen-Probe & HPLC & & & & & \\
\hline 55 & M. avium & M. avium & M. intracellulare & 2 & None & $<30$ & 0 & + \\
\hline 54 & M. avium & M. avium & M. avium & 4 & None & $>45$ & 1 & - \\
\hline 16 & M. avium & M. avium & M. avium & 6 & Pale yellow & $>45$ & 1 & - \\
\hline 5 & $M$. intracellulare & $M$. intracellulare & M. intracellulare & 7 & Orange & 34 & 0 & - \\
\hline 33 & M. scrofulaceum & $\mathrm{NRX}^{b}$ & M. scrofulaceum & 7 & Yellow & $>45$ & 0 & - \\
\hline 29 & M. avium & M. avium & M. avium & 8 & None & $>45$ & 1 & - \\
\hline 3 & $M$. intracellulare & M. intracellulare & M. intracellulare & 14 & Orange & $>45$ & 0 & - \\
\hline 2 & $\mathrm{NT}^{c}$ & NRX & $M$. intracellulare & 18 & None & $<30$ & 0 & - \\
\hline 8 & M. intracellulare & $M$. intracellulare & M. intracellulare & 19 & None & $>45$ & 0 & - \\
\hline 42 & M. scrofulaceum & NRX & M. avium & 22 & Orange & 40 & 0 & - \\
\hline 58 & $M$. intracellulare & NRX & $?$ & 22 & None & $?$ & $?$ & $?$ \\
\hline 37 & $M$. intracellulare & NRX & M. intracellulare & 23 & None & $<30$ & 0 & - \\
\hline 51 & M. intracellulare & NRX & M. intracellulare & 23 & None & $>45$ & 0 & - \\
\hline 39 & M. scrofulaceum & NRX & M. scrofulaceum & 26 & Orange & $>45$ & 0 & - \\
\hline 43 & $M$. intracellulare & NRX & M. scrofulaceum & 28 & None & $>45$ & 0 & - \\
\hline
\end{tabular}

a 0 , no growth; 1 , growth; ?, questionable growth.

${ }^{b}$ NRX, no reaction with any of the probes tested.

$c$ NT, test not performed.

with the nominal serovar assignments of the original 62 strains tested, discrepancies were seen with strains 21,24 , 25 , and 51 . In three of these cases, the results of the confirmatory analysis in laboratory 7 were in agreement with the consensus results of laboratories 1,3 , and 5, suggesting that there was a labelling error in the reference stock collection; no consensus emerged from the results obtained with strain 25 . Workers in laboratory 7 , who used a combination of seroagglutination, ELISA, and chromatography, had only one disagreement with the consensus serovar results for the 61 results which they submitted, for an agreement rate of $98.5 \%$. For laboratory 1 , the only laboratory that routinely confirmed agglutination results with absorption tests, the rate of agreement with the consensus results was $88.7 \%$; four of the seven failures were associated with spontaneous agglutination of the cell suspensions, which interfered with the reading of results. For laboratory 3 the rate of agreement with the consensus results was $71 \%$; only one of the disagreements was due to spontaneous agglutination, and the remainder were equally divided between lack of agglutination with any of the test sera and agglutination with a serum that did not conform to the consensus serovar. For laboratory 5 the rate of agreement with consensus results was $93.4 \%$; two disagreements were associated with spontaneous agglutination, and the other two were associated with failure to agglutinate with any of the sera.

Laboratories 2, 4, and 6 each lacked antisera to a number of the serovars represented in this study and would have been expected to report many strains as not typeable in their systems. In addition to reporting a large number of strains as unidentifiable because of missing sera, these laboratories also tended to ascribe a disproportionate number of these strains to the wrong serovars, presumably because of unrecognized cross-reactions.

\section{DISCUSSION}

The data in Table 2 support the redistribution of serovars among the species $M$. intracellulare and $M$. avium that was suggested previously by the results of independent DNA hybridization tests (1), Gen-Probe studies (15), and T-cata- lase serologic studies $(19,20)$. They also illustrate the caution that must be exercised in assuming that a member of a particular serovar automatically must belong to a certain species. In this study in at least four instances (serovars 7 , 18,26 , and 43 and possibly serovar 22 ), two or more strains belonging to a particular serovar were placed in two different species according to the semantide-based species assignment criteria used. However, despite these few exceptions, the common ascription of serovars 1 to 6 and 8 to 11 to $M$. avium and serovars 7 and 12 to 20 to $M$. intracellulare is supported by the data presented here. Furthermore, these data provide a basis for ascribing members of serovar 21 to $M$. avium and members of serovars 26 and 27 to $M$. scrofulaceum; the last two ascriptions are supported by similar conclusions of Saito and his colleagues, which were based on an examination of these strains with antibody specific for the alpha antigen of $M$. scrofulaceum (15).

The strains of serovars 22 and 23 that appeared to fall into the $M$. avium complex on the basis of molecular and cultural criteria, but failed to react with the DNA probe for either $M$. avium or $M$. intracellulare, appear to correspond to organisms belonging to cluster 4 as described in a recent openended study report of the IWGMT (28). Similar strains have been reported to react with the SNAP X probe (Syngene, Inc., San Diego, Calif.) (11). The T-catalase serologic results, which reflect mRNAs, and the results of recent rRNA analyses performed by Ferguson and colleagues (6) suggest that these organisms may be closely related to $M$. intracellulare. These and similar strains are under more intensive investigation in a current semantide-based IWGMT study.

In laboratories 1,3 , and 5, which relied on agglutination of the coded strains with a full panel of sera, the mean level of agreement with the consensus serovar results was $84 \%$; 7 of the 30 discrepancies were associated with spontaneous agglutination. The use of an ELISA or chromatography of extracts might have permitted determination of the serovars for those strains $(5,17)$. The highest proportion of reactions of individual strains with more than one serum occurred in laboratory 1 , and the specificity of those reactions was supported by the results of absorption tests. 
The high proportion of strains that were ascribed to nonconsensus serovars in laboratories 2,4 , and 6 , which did not use a full panel of sera for all of the serovars represented in this study, may have been associated with the use of antiserum concentrations that were too high, allowing crossreactions to occur. Sera that are prepared by inoculation of rabbits with whole cells of serovar reference strains contain some antibody to the common mycobacterial antigens that lie at the bacterial surface, underneath the loose coat of the glycopeptidolipid antigen responsible for serovar-specific agglutination (29). These results illustrate the value of using preabsorbed sera and confirming agglutination results by absorption with the test strain, especially when less than a full panel of sera is used.

The results of this study validate the utility of most of the existing reference serovar strains but identify possible problems with strains 21 (= ATCC 23435), 24 (= 6845), 25 (= AT 545 Findley), and 51 (= 12645).

\section{REFERENCES}

1. Baess, I. 1983. Deoxyribonucleic acid relationships between different serovars of Mycobacterium avium, Mycobacterium intracellulare and Mycobacterium scrofulaceum. Acta Pathol. Microbiol. Immunol. Scand. 91:201-203.

2. Brennan, P. J., H. Mayer, G. O. Aspinall, and J. E. Nam Shin. 1981. Structures of the glycopeptidolipid antigens from serovars in the Mycobacterium avium/Mycobacterium intracellulare/Mycobacterium scrofulaceum serocomplex. Eur. J. Biochem. 115: 7-15.

3. Butler, W. R., K. C. Jost, Jr., and J. O. Kilburn. 1991. Identification of mycobacteria by high-performance liquid chromatography. J. Clin. Microbiol. 29:2468-2472.

4. Butler, W. R., and J. O. Kilburn. 1988. Identification of major slowly growing pathogenic mycobacteria and Mycobacterium gordonae by high-performance liquid chromatography of their mycolic acids. J. Clin. Microbiol. 26:50-53.

5. Denner, J. C., A. Y. Tsang, D. Chatterjee, and P. J. Brennan. 1992. Comprehensive approach to identification of serovars of Mycobacterium avium complex. J. Clin. Microbiol. 30:473-478.

6. Ferguson, C., P. Hammond, R. Lankford, D. McAllister, and V. Jonas. 1991. Phenotypic discordance within species of the genus Mycobacterium revealed by application of DNA probes, abstr. 30. Progr. Abstr. 91st Annu. Meet. Am. Soc. Microbiol. 1991. American Society for Microbiology, Washington, D.C.

7. Good, R. C., and R. E. Beam. 1984. Seroagglutination, p. 105-122. In G. P. Kubica and L. G. Wayne (ed.), The mycobacteria: a sourcebook. Marcel Dekker, Inc., New York.

8. Goodfellow, M., A. Lind, H. Mordarska, S. Pattyn, and M. Tsukamura. 1974. A co-operative numerical analysis of cultures considered to belong to the "rhodochrous" taxon. J. Gen. Microbiol. 85:291-302.

9. Goslee, S., T. K. Rynearson, and E. Wolinsky. 1976. Additional serotypes of Mycobacterium scrofulaceum, Mycobacterium gordonae, Mycobacterium marinum, and Mycobacterium xenopi determined by agglutination. Int. J. Syst. Bacteriol. 26: 136-142.

10. Kubica, G. P., I. Baess, R. E. Gordon, P. A. Jenkins, J. B. G. Kwapinski, C. McDurmont, S. R. Pattyn, H. Saito, V. A. Silcox, J. L. Stanford, K. Takeya, and M. Tsukamura. 1972. A cooperative numerical analysis of rapidly growing mycobacteria. $\mathrm{J}$. Gen. Microbiol. 73:55-70.

11. Lim, S. D., J. Todd, J. Lopez, E. Ford, and J. M. Janda. 1991. Genotypic identification of pathogenic Mycobacterium species by using a nonradioactive oligonucleotide probe. J. Clin. Microbiol. 29:1276-1278.

12. Meissner, G., K. H. Schroder, G. E. Amadio, W. Anz, S. Chaparas, H. W. B. Engel, P. A. Jenkins, W. Kappler, H. H. Kleeberg, E. Kubala, M. Kubín, D. Lauterbach, A. Lind, M. Magnusson, Z. D. Mikova, S. R. Pattyn, W. B. Schaefer, J. L. Stanford, M. Tsukamura, L. G. Wayne, I. Willers, and E. Wolinsky. 1974. A cooperative numerical analysis of nonscoto- and nonphotochromogenic slowly growing mycobacteria. J. Gen. Microbiol. 83:207-235.

13. Reznikov, M., and J. H. Leggo. 1972. Modification of Schaefer's procedure for serotyping of organisms of the Mycobacterium avium-M. intracellulare-M. scrofulaceum complex. Appl. Microbiol. 23:819-823.

14. Saito, H., R. E. Gordon, I. Juhlin, W. Käppler, J. B. G. Kwapinski, C. McDurmont, S. R. Pattyn, E. H. Runyon, J. L. Stanford, I. Tarnok, H. Tasaka, M. Tsukamura, and J. Weiszfeiler. 1977. Cooperative numerical analysis of rapidly growing mycobacteria. Int. J. Syst. Bacteriol. 27:75-85.

15. Saito, H., H. Tomioka, K. Sato, H. Tasaka, and D. J. Dawson. 1990. Identification of various serovar strains of Mycobacterium avium complex by using DNA probes specific for Mycobacterium avium and Mycobacterium intracellulare. J. Clin. Microbiol. 28:1694-1697.

16. Schaefer, W. B. 1965 . Serologic identification and classification of atypical mycobacteria by their agglutination. Am. Rev. Respir. Dis. 92(Suppl.):85-93.

17. Tsang, A. Y., J. C. Denner, P. J. Brennan, and J. K. McClatchy. 1992. Clinical and epidemiological importance of typing of Mycobacterium avium complex isolates. J. Clin. Microbiol. 30:479-484.

18. Wayne, L. G., L. Andrade, S. Froman, W. Kappler, E. Kubala, G. Meissner, and M. Tsukamura. 1979. A co-operative numerical analysis of Mycobacterium gastri, Mycobacterium kansasii, and Mycobacterium marinum. J. Gen. Microbiol. 109:319-327.

19. Wayne, L. G., and G. A. Diaz. 1986. Differentiation between T-catalases derived from Mycobacterium avium and Mycobacterium intracellulare by a solid-phase immunosorbent assay. Int. J. Syst. Bacteriol. 36:363-367.

20. Wayne, L. G., and G. A. Diaz. 1987. Intrinsic catalase dot blot immunoassay for identification of Mycobacterium tuberculosis, Mycobacterium avium, and Mycobacterium intracellulare. J. Clin. Microbiol. 25:1687-1690.

21. Wayne, L. G., T. M. Dietz, C. Gernez-Rieux, P. A. Jenkins, W. Käppler, G. P. Kubica, J. B. G. Kwapinski, G. Meissner, S. R. Pattyn, E. H. Runyon, K. H. Schröder, V. A. Silcox, M. Tsukamura, and E. Wolinsky. 1971. A cooperative numerical taxonomic analysis of scotochromogenic slowly growing mycobacteria. J. Gen. Microbiol. 66:255-271.

22. Wayne, L. G., H. C. Engbaek, H. W. B. Engel, S. Froman, W. Gross, J. Hawkins, W. Käppler, A. G. Karlson, H. H. Kleeberg, I. Krasnow, G. P. Kubica, C. McDurmont, E. E. Nel, S. R. Pattyn, K. H. Schröder, S. Showalter, I. Tarnok, M. Tsukamura, B. Vergmann, and E. Wolinsky. 1974. Highly reproducible techniques for use in systematic bacteriology in the genus Mycobacterium: tests for pigment, urease, resistance to sodium chloride, hydrolysis of Tween 80 , and $\beta$-galactosidase. Int. J. Syst. Bacteriol. 24:412-419.

23. Wayne, L. G., H. W. B. Engel, C. Grassi, W. Gross, J. Hawkins, P. A. Jenkins, W. Käppler, H. H. Kleeberg, I. Krasnow, E. E. Nel, S. R. Pattyn, P. A. Richards, S. Showalter, M. Slosarek, I. Szabo, I. Tarnok, M. Tsukamura, B. Vergmann, and E. Wolinsky. 1976. Highly reproducible techniques for use in systematic bacteriology in the genus Mycobacterium. II. Tests for niacin and catalase and for resistance to isoniazid, thiophene 2-carboxylic acid hydrazide, hydroxylamine, and $p$-nitrobenzoate. Int. J. Syst. Bacteriol. 26:311-318.

24. Wayne, L. G., R. C. Good, M. I. Krichevsky, R. E. Beam, Z. Blacklock, S. D. Chaparas, D. Dawson, S. Froman, W. Gross, J. Hawkins, P. A. Jenkins, I. Juhlin, W. Käppler, H. H. Kleeberg, I. Krasnow, M. J. Lefford, E. Mankiewicz, C. McDurmont, G. Meissner, P. Morgan, E. E. Nel, S. R. Pattyn, F. Portaels, P. A. Richards, S. Rüsch, K. H. Schröder, V. A. Silcox, I. Szabo, M. Tsukamura, and B. Vergmann. 1981. First report of the cooperative, open-ended study of slowly growing mycobacteria by the International Working Group on Mycobacterial Taxonomy. Int. J. Syst. Bacteriol. 31:1-20.

25. Wayne, L. G., R. C. Good, M. I. Krichevsky, R. E. Beam, Z. Blacklock, H. L. David, D. Dawson, W. Gross, J. Hawkins, P. A. Jenkins, I. Juhlin, W. Käppler, H. H. Kleeberg, I. Krasnow, M. J. Lefford, E. Mankiewicz, C. McDurmont, E. E. Nel, F. 
Portaels, P. A. Richards, S. Rüsch, K. H. Schröder, V. A. Silcox, I. Szabo, M. Tsukamura, L. Van den Breen, and B. Vergmann. 1983. Second report of the cooperative, open-ended study of slowly growing mycobacteria by the International Working Group on Mycobacterial Taxonomy. Int. J. Syst. Bacteriol. 33:265-274.

26. Wayne, L. G., R. C. Good, M. I. Krichevsky, Z. Blacklock, H. L. David, D. Dawson, W. Gross, J. Hawkins, P. A. Jenkins, I. Juhlin, W. Käppler, H. H. Kleeberg, V. Levy-Frebault, C. McDurmont, E. E. Nel, F. Portaels, S. Rüsch-Gerdes, K. H. Schröder, V. A. Silcox, I. Szabo, M. Tsukamura, L. Van Den Breen, B. Vergmann, and M. A. Yakrus. 1989. Third report of the cooperative, open-ended study of slowly growing mycobacteria by the International Working Group on Mycobacterial Taxonomy. Int. J. Syst. Bacteriol. 39:267-278.

27. Wayne, L. G., R. C. Good, M. I. Krichevsky, Z. Blacklock, H. L. David, D. Dawson, W. Gross, J. Hawkins, V. V. Levy-Frebault,
C. McManus, F. Portaels, S. Rüsch-Gerdes, K. H. Schröder, V. A. Silcox, M. Tsukamura, L. Van Den Breen, and M. A. Yakrus. 1991. Fourth report of the cooperative open-ended study of slowly growing mycobacteria by the International Working Group on Mycobacterial Taxonomy. Int. J. Syst. Bacteriol. 41:463-472.

28. Wayne, L. G., D. Hollander, B. Anderson, H. A. Sramek, C. M. Vadheim, and J. I. Rotter. 1992. Immunoglobulin A (IgA) and IgG serum antibodies to mycobacterial antigens in Crohn's disease patients and their relatives. J. Clin. Microbiol. 30:20132018.

29. Wolinsky, E. 1979. Emendation of proposed additional serotypes of mycobacteria determined by agglutination. Int. J. Syst. Bacteriol. 29:59.

30. Wolinsky, E., and W. B. Schaefer. 1973. Proposed numbering scheme for mycobacterial serotypes. Int. J. Syst. Bacteriol. 23:182-183. 